\title{
Hubble Space Telescope Angular Velocity Estimation During the Robotic Servicing Mission
}

\author{
Julie K. Thienel*, Steven Z. Queen ${ }^{\dagger}$, and John M. VanEepoel ${ }^{\ddagger}$ \\ NASA Goddard Space Flight Center, Flight Dynamics Analysis Branch \\ Greenbelt, Maryland, 20771, USA \\ Robert M. Sanner ${ }^{\S}$ \\ University of Maryland, Aerospace Engineering Department, College Park, Maryland, 20742, USA
}

\begin{abstract}
In 2004 NASA began investigation of a robotic servicing mission for the Hubble Space Telescope (HST). Such a mission would require estimates of the HST attitude and rates in order to achieve a capture by the proposed Hubble robotic vehicle (HRV). HRV was to be equipped with vision-based sensors, capable of estimating the relative attitude between HST and HRV. The inertial HST attitude is derived from the measured relative attitude and the HRV computed inertial attitude. However, the relative rate between HST and HRV cannot be measured directly. Therefore, the HST rate with respect to inertial space is not known. Two approaches are developed to estimate the HST rates. Both methods utilize the measured relative attitude and the HRV inertial attitude and rates. First, a nonlinear estimator is developed. The nonlinear approach estimates the HST rate through an estimation of the inertial angular momentum. Second, a linearized approach is developed. The linearized approach is a pseudo-linear Kalman filter. Simulation test results for both methods are given. Even though the development began as an application for the HST robotic servicing mission, the methods presented are applicable to any rendezvous/capture mission involving a non-cooperative target spacecraft.
\end{abstract}

\section{Introduction}

The Hubble Space Telescope (HST) was launched in 1990 and has undergone four servicing missions throughout its mission lifetime to replace instruments, sensors, solar arrays, power units, and cooling systems. Additional servicing will again be necessary to extend HST's science life. The batteries are predicted to fail as early as 2009 , and the pointing control system may be reduced to a two-gyro mode by as early as 2007 . On March 12, 2004 former NASA Administrator Sean O'Keefe asked the HST program to investigate robotic servicing of the HST to extend the science life. In addition to replacing the batteries and gyros, the servicing mission would install two new instruments and would provide the ability to safely de-orbit HST.

The original robotic servicing mission concept included two vehicles, a de-orbit module (DM) and an ejection module (EM) with the robotic arm for servicing. The DM docks with HST and provides batteries and gyros for the stacked HST-DM configuration. The DM also provides the reentry capability for the stacked configuration. The EM carries the robotic arm for servicing, as well as the replacement instruments. Following the servicing, the EM separates from the HST-DM stack and safely de-orbits. An alternative concept to the robotic servicing mission was to provide a de-orbit only capability for HST. A modified DM docks with HST and provides for a safe re-entry of HST. In this work we refer to the vehicle docking with HST as the Hubble Robotic Vehicle (HRV).

In order for the HRV to dock with HST, the HRV must match the rotation rates of HST. If the batteries and gyros both fail, HST will be tumbling with unknown rates. The HRV will be equipped with sensors

\footnotetext{
*Aerospace Engineer, julie.thienel@nasa.gov, phone:301-286-9033, and AIAA Member.

${ }^{\dagger}$ Aerospace Engineer, steven.z.queen@nasa.gov, phone:301-286-0988.

$\ddagger$ Aerospace Engineer, john.m. vaneepoel@nasa.gov, phone:301-286-9216.

${ }^{\S}$ Professor of Aerospace Engineering, rmsanner@eng.umd.edu, phone:301-405-1928, and AIAA member.
} 
capable of estimating the relative attitude between HST and HRV. The sensors utilize vision and feature recognition measurements to produce a relative quaternion. The HRV will also be equipped with star cameras and gyros which will provide the inertial orientation and body rates of the HRV. Combining the relative attitude and HRV inertial attitude provides a measurement of the HST attitude. However, the relative rates or HST body rates cannot be measured directly.

We present and compare two approaches for estimating the HST body rates in the event that HST is tumbling and no a priori rate information is available. First a nonlinear estimator is developed. The nonlinear approach determines the HST rate through an estimation of the inertial angular momentum. The stability properties of the estimator are addressed first for perfect attitude measurements. Errors are then introduced into the HST attitude. The primary error source considered is the uncertainty in the relative attitude quaternion provided by the vision and feature recognition sensors. The results of the nonlinear approach are then compared to a pseudo-linear Kalman filter.

Even though this work was originally developed for the HST/HRV scenario, it can easily be applied to other 'non-cooperative' rendezvous/capture scenarios. A 'non-cooperative' scenario is one in which the body rates of the target vehicle are not available, and the target vehicle does not carry any devices to improve the relative attitude estimation, such as reflectors or visible beacons.

The paper is outlined as follows. The next section presents definitions and mathematical background. Section III presents the nonlinear estimation algorithm. Section IV discusses how measurement errors affect the nonlinear algorithm. Section V presents an overview of the pseudo-linear Kalman filter. Section VI includes results, followed by conclusions in the last section.

\section{Attitude and Angular Rate Definitions}

The attitude of a spacecraft can be represented by a quaternion, consisting of a rotation angle and unit rotation vector $e$, known as the Euler axis, and a rotation $\phi$ about this axis so that ${ }^{1}$

$$
\boldsymbol{q}=\left[\begin{array}{c}
e \sin \left(\frac{\phi}{2}\right) \\
\cos \left(\frac{\phi}{2}\right)
\end{array}\right]=\left[\begin{array}{l}
\varepsilon \\
\eta
\end{array}\right]
$$

where $\boldsymbol{q}$ is the quaternion, partitioned into a vector part, $\varepsilon$, and a scalar part, $\eta$. The Hubble Space Telescope (HST) attitude quaternion is designated as $\boldsymbol{q}_{v}$, which defines the rotation from inertial to HST body coordinates. The Hubble Robotic Vehicle (HRV) attitude quaternion is designated as $\boldsymbol{q}_{h}$.

The rotation, or attitude, matrix can be computed from the quaternion components as ${ }^{1}$

$$
R=R(\boldsymbol{q})=\left(\eta^{2}-\varepsilon^{T} \varepsilon\right) \mathrm{I}_{3}+2 \varepsilon \varepsilon^{T}-2 \eta S(\varepsilon)
$$

where $\mathrm{I}_{3}$ is a $3 \times 3$ identity matrix and $S(\varepsilon)$ is a matrix representation of the vector cross product operation.

$$
S(\varepsilon)=\left[\begin{array}{ccc}
0 & -\varepsilon_{z} & \varepsilon_{y} \\
\varepsilon_{z} & 0 & -\varepsilon_{x} \\
-\varepsilon_{y} & \varepsilon_{x} & 0
\end{array}\right]
$$

Note also that $R(q) \varepsilon=\varepsilon$

A relative rotation between coordinate frames is computed $a s^{2}$

$$
\tilde{\boldsymbol{q}}=\left[\begin{array}{c}
\tilde{\boldsymbol{\varepsilon}} \\
\tilde{\eta}
\end{array}\right]=\boldsymbol{q}_{1} \otimes \boldsymbol{q}_{2}^{-1}=\left[\begin{array}{cc}
\eta_{2} I-S\left(\varepsilon_{2}\right) & -\varepsilon_{2} \\
\epsilon_{2}^{T} & \eta_{2}
\end{array}\right]\left[\begin{array}{l}
\varepsilon_{1} \\
\eta_{1}
\end{array}\right]
$$

Using the definition given in equation 3 , the relative attitude quaternion from HRV body coordinates to HST body coordinates is then

$$
q_{r}=q_{v} \otimes q_{h}^{-1}
$$

The angular velocity of the HST body coordinates with respect to inertial space, resolved in HST body coordinates, is designated as $\omega_{v}$. Similarly the angular velocity of the HRV in HRV body coordinates is designated as $\omega_{h}$. The angular velocity of HST relative to HRV, given in HST body coordinates, is given as

$$
\omega_{r}^{v}=\omega_{v}-R_{r} \omega_{h}
$$

where $R_{r}$ is the relative attitude matrix which transforms $\omega_{h}$ into HST body coordinates. 


\section{HST Nonlinear Angular Velocity Estimator}

The following angular velocity estimator is intended for the scenario in which the HST batteries have died. No telemetry is available from HST. HRV is equipped with a quaternion star tracker, which gives $q_{h}$, and some sensor system which produces the relative quaternion, $\boldsymbol{q}_{r}$. The approach does not yet account for any errors in the measured quaternions, the HST inertia matrix, or the computation of the external torques on HST. The HST angular velocity is estimated in the inertial coordinate system through the estimation of the inertial angular momentum. The HST angular velocity in body coordinates is computed by a transformation of the inertial angular velocity. In the following developments, the time argument is omitted to simplify the notation.

The angular momentum in inertial coordinates is defined as

$$
h_{i, v}=I_{i, v} \omega_{i, v}
$$

where $I_{i, v}$ is the HST inertia matrix and $\omega_{\boldsymbol{i}, \boldsymbol{v}}$ is the angular velocity, both in inertial coordinates. Note that $I_{i, v}=R_{v}^{T} I_{v} R_{v}$, where $R_{v}$ is the HST attitude matrix defining the transformation from inertial to HST body coordinates. $I_{v}$ is the HST inertia matrix in body coordinates, assumed to be constant. The kinematic equation for the quaternion is given as

$$
\dot{\boldsymbol{q}}_{v}=\frac{1}{2} Q\left(\boldsymbol{q}_{v}\right) \boldsymbol{\omega}_{v}=\frac{1}{2} Q\left(\boldsymbol{q}_{v}\right) R_{v} \boldsymbol{\omega}_{i, v}=\frac{1}{2} Q\left(\boldsymbol{q}_{v}\right) I_{v}^{-1} R_{v} \boldsymbol{h}_{i, v}
$$

where

$$
Q\left(\boldsymbol{q}_{v}\right)=\left[\begin{array}{c}
\eta_{v} \mathrm{I}_{3}+S\left(\varepsilon_{v}\right) \\
-\varepsilon_{v}^{T}
\end{array}\right]=\left[\begin{array}{c}
Q_{1}\left(\boldsymbol{q}_{v}\right) \\
-\varepsilon_{v}^{T}
\end{array}\right]
$$

where, by inspection, $Q_{1}\left(\boldsymbol{q}_{v}\right)=\eta_{v} \mathrm{I}_{3}+S\left(\varepsilon_{v}\right)$. The kinematic equation for the attitude matrix is ${ }^{3}$

$$
\dot{R}_{v}=-S\left(\omega_{v}\right) R_{v}
$$

Note that the $\boldsymbol{q}_{v}$ (and therefore the attitude matrix $R_{v}$ ) is supplied by the HRV measured inertial attitude and the measured relative attitude. Using equation 4

$$
\boldsymbol{q}_{v}=\boldsymbol{q}_{\boldsymbol{r}} \otimes \boldsymbol{q}_{h}
$$

Similarly

$$
R_{v}=R_{r} R_{h}
$$

Euler's equation describes the dynamics of a rigid spacecraft. Euler's equation for HST is given in inertial coordinates as ${ }^{4}$

$$
\dot{h}_{i, v}=T_{i, v}
$$

$T_{i, v}$ is the external torque acting on HST, resolved in inertial coordinates.

Define the estimated angular momentum as

$$
\hat{\boldsymbol{h}}_{i, v}=I_{i, v} \hat{\boldsymbol{\omega}}_{i, v}
$$

where $\hat{\omega}_{i, v}$ is the estimated angular velocity in inertial coordinates. The estimated angular velocity in body coordinates is then

$$
\hat{\boldsymbol{\omega}}_{v}=R_{v} \hat{\boldsymbol{\omega}}_{i, v}=R_{v} I_{i, v}^{-1} \hat{\boldsymbol{h}}_{i, v}=I_{v}^{-1} R_{v} \hat{\boldsymbol{h}}_{i, v}
$$

Using equation 1 , define the predicted HST quaternion as

$$
\hat{\boldsymbol{q}}_{v}=\left[\begin{array}{l}
\hat{\varepsilon}_{v} \\
\hat{\eta}_{v}
\end{array}\right]
$$

The attitude error is defined as the relative orientation between the predicted attitude $\hat{\boldsymbol{q}}_{v}$ and the attitude provided by the measured attitude, $\boldsymbol{q}_{v}$. The attitude error is

$$
\tilde{\boldsymbol{q}}_{v}=\left[\begin{array}{l}
\tilde{\varepsilon}_{\boldsymbol{v}} \\
\tilde{\eta}_{v}
\end{array}\right]=\boldsymbol{q}_{v} \otimes \hat{\boldsymbol{q}}_{v}^{-1}
$$


Following the estimators proposed in Refs. 5,6, and 4, a state estimator for the HST attitude and angular momentum is defined as

$$
\begin{gathered}
\dot{\hat{\boldsymbol{q}}}_{v}=\frac{1}{2} Q\left(\hat{\boldsymbol{q}}_{v}\right) R\left(\overline{\boldsymbol{q}}_{v}(t)\right)^{T}\left[I_{v}^{-1} R_{v} \hat{\boldsymbol{h}}_{i, v}+k \tilde{\varepsilon}_{v} \operatorname{sign}\left(\tilde{\eta}_{v}\right)\right] \\
\dot{\hat{\boldsymbol{h}}}_{i, v}=T_{i, v}+\frac{\alpha}{2} R_{v}^{T} I_{v}^{-1} \tilde{\epsilon}_{v} \operatorname{sign}\left(\tilde{\eta}_{v}\right)
\end{gathered}
$$

The term $R\left(\tilde{\boldsymbol{q}}_{v}(t)\right)^{T}$ in equation 13 transforms the angular velocity terms from the body frame to the predicted attitude frame. The gain $\mathrm{k}$ is chosen as a positive constant. Similarly, the learning rate, $\alpha$, is also a positive constant. Essentially, $\hat{\boldsymbol{q}}_{v}$ is a prediction of the attitude at time $t$, propagated with the kinematic equation using the estimated angular momentum.

The kinematic equation for the attitude error quaternion, $\tilde{q}_{v}$, has the same form as the quaternion kinematic equation in equation 6 . The angular velocity associated with the attitude error quaternion is the difference between the angular velocity of the body coordinates and the angular velocity of the estimator coordinates (resolved in body coordinates). ${ }^{7}$ Therefore, with equation 6 , the definition given in equation 7 , equation 13 , and noting that $R\left(\tilde{\boldsymbol{q}}_{v}(t)\right) \tilde{\varepsilon}_{v}=\tilde{\varepsilon}_{v}$ (since $\tilde{\varepsilon}_{v}$ points along the eigenaxis of the rotation), the kinematic equation for $\tilde{\boldsymbol{q}}_{v}$ is given as

$$
\dot{\boldsymbol{q}}_{v}=\frac{1}{2}\left[\begin{array}{c}
Q_{1}\left(\tilde{\boldsymbol{q}}_{v}\right) \\
-\tilde{\boldsymbol{\varepsilon}}_{v}^{T}
\end{array}\right]\left(I_{v}^{-1} R_{v} \boldsymbol{h}_{i, v}-I_{v}^{-1} R_{v} \hat{\boldsymbol{h}}_{i, v}-k \tilde{\boldsymbol{\varepsilon}}_{v} \operatorname{sign}\left(\tilde{\eta}_{v}\right)\right.
$$

Let $\tilde{\boldsymbol{h}}_{i, v}=\boldsymbol{h}_{i, v}-\hat{\boldsymbol{h}}_{i, v}$. The derivative of $\tilde{\boldsymbol{h}}_{i, v}$ is

$$
\dot{\tilde{\boldsymbol{h}}}_{i, v}=-\frac{\alpha}{2} R_{v}^{T} I_{v}^{-1} \tilde{\varepsilon}_{v} \operatorname{sign}\left(\tilde{\eta}_{v}\right)
$$

Note that the equilibrium states for 15 and 16 are

$$
\left[\begin{array}{ll}
\tilde{\boldsymbol{q}}_{v}^{\boldsymbol{T}} & \tilde{\boldsymbol{h}}_{\boldsymbol{i}, v}^{\boldsymbol{T}}
\end{array}\right]=\left[\begin{array}{lllllll}
0 & 0 & 0 & \pm 1 & 0 & 0 & 0
\end{array}\right]
$$

Proof of stability: Choose a Lyapunov function as (note that the time argument is omitted from the variables on the right side of the equation for clarity)

$$
V(t)=\frac{1}{2 \alpha} \tilde{h}_{i, v}^{T} \tilde{h}_{i, v}+\frac{1}{2} \begin{cases}\left(\tilde{\eta}_{v}-1\right)^{2}+\tilde{\varepsilon}_{v}^{T} \tilde{\varepsilon}_{v} & \tilde{\eta}_{v} \geq 0 \\ \left(\tilde{\eta}_{v}+1\right)^{2}+\tilde{\varepsilon}_{v}^{T} \tilde{\varepsilon}_{v} & \tilde{\eta}_{v}<0\end{cases}
$$

$V(t)$ is continuous. The derivative of $V(t)$ is

$$
\dot{V}(t)=\frac{1}{\alpha} \tilde{h}_{i, v}^{T} \dot{\tilde{h}}_{i, v}+ \begin{cases}\left(\tilde{\eta}_{v}-1\right) \dot{\tilde{\eta}}_{o}+\tilde{\varepsilon}_{v}^{T} \dot{\tilde{\varepsilon}}_{o} & \tilde{\eta}_{v} \geq 0 \\ \left(\tilde{\eta}_{v}+1\right) \dot{\tilde{\eta}}_{o}+\tilde{\varepsilon}_{v}^{T} \dot{\tilde{\varepsilon}}_{o} & \tilde{\eta}_{v}<0\end{cases}
$$

Noting that $\tilde{\varepsilon}_{v}^{T} \dot{\vec{\varepsilon}}_{o}+\tilde{\eta}_{v} \dot{\tilde{\eta}}_{o}=0$ (including the left and right derivatives of $\tilde{\eta}_{v}=0$ ), for all $\mathrm{t}$, equation 17 is

$$
\dot{V}(t)=-\frac{k}{2} \widetilde{\varepsilon}_{v}^{T} \tilde{\varepsilon}_{v}
$$

This establishes that $\tilde{h}_{i, v}, \tilde{\varepsilon}_{v}$, and $\tilde{\eta}_{v}$ are globally, uniformly bounded. Moreover, $V(t)$ is a continuous, twice differentiable function with

$$
\ddot{V}_{o}(t)=-\frac{k}{2} \bar{\varepsilon}_{v}^{T} Q_{1}\left(\tilde{\boldsymbol{q}}_{v}\right)\left[I_{v}^{-1} R_{v} \tilde{h}_{i, v}-k \tilde{\varepsilon}_{v} \operatorname{sign}\left(\tilde{\eta}_{v}\right)\right]
$$

which is bounded. Barbalat's lemma then shows that $\left\|\tilde{\varepsilon}_{v}\right\| \rightarrow 0$ as $t \rightarrow \infty .^{8}$

Since all signals in the estimator are bounded, the system 15 and 16 can be further analyzed, in the given format, as a linear time-varying system ${ }^{9} \dot{\boldsymbol{x}}(t)=A(t) \boldsymbol{x}(t)$ where

$$
\boldsymbol{x}(t)=\left[\begin{array}{c}
\tilde{\varepsilon}_{v} \\
\tilde{\boldsymbol{h}}_{i, v}
\end{array}\right]
$$




$$
A(t)=\left[\begin{array}{cc}
-\frac{k}{2} \operatorname{sign}\left(\tilde{\eta}_{v}\right) Q_{1}\left(\tilde{\boldsymbol{q}}_{v}\right) & \frac{1}{2} Q_{1}\left(\tilde{\boldsymbol{q}}_{v}\right) I_{v}^{-1} R_{v} \\
\frac{\alpha}{2} \operatorname{sign}\left(\tilde{\eta}_{v}\right) R_{v}^{T} I_{v}^{-1} & 0
\end{array}\right]
$$

where, by virtue of the above Lyapunov analysis, all terms in the matrix $A(t)$ are known to be bounded for all $t \geq t_{0}$. Rewriting $\dot{V}(t)$ as $\dot{V}(t)=-\boldsymbol{x}(t)^{T} C^{T} C \boldsymbol{x}(t) \leq 0$, where $C=\left[\begin{array}{ll}\sqrt{\frac{k}{2}} \mathrm{I}_{3} & 0\end{array}\right]$, Theorem 4.5 and the discussion on pp.626-628 in Ref. 9 shows that the equilibrium point $\boldsymbol{x}(t)=0$ of this equivalent system is exponentially stable if the pair $(A(t), C)$ is uniformly completely observable (UCO). Since observability properties are unchanged under output feedback, ${ }^{9}(A(t), C)$ are UCO if the pair $(A(t)-K(t) C, C)$ is uniformly observable for any piecewise, continuous and bounded matrix $K(t)$. Choose $K(t)$ as

$$
K(t)=\left[\begin{array}{c}
-\sqrt{\frac{k}{2}} \operatorname{sign}\left(\tilde{\eta}_{v}\right) Q_{1}\left(\tilde{\boldsymbol{q}}_{v}\right) \\
\sqrt{\frac{\alpha}{2 k}} \operatorname{sign}\left(\tilde{\eta}_{v}\right) R_{v}^{T} I_{v}^{-1}
\end{array}\right]
$$

$K(t)$ is piecewise continuous based on the following properties. Note from the above Lyapunov analysis that $\left\|\tilde{\varepsilon}_{v}\right\| \rightarrow 0$. Since $\left\|\tilde{\boldsymbol{q}}_{v}\right\|^{2}=1=\left\|\tilde{\varepsilon}_{v}\right\|^{2}+\left|\tilde{\eta}_{v}\right|^{2}$ for any time, $t$, there exists a time, $T>0$, such that $\left\|\tilde{\eta}_{v}\right\|>0$ for all $t>T$. Since $\tilde{\eta}_{v}$ therefore cannot pass through zero for $t>T$, $\operatorname{sign}\left(\tilde{\eta}_{v}\right)$ is constant for all $t>T$ and, hence, $K(t)$ is a piecewise continuous function of time.

The state transition matrix for the pair $(A(t)-K(t) C, C)$ is

$$
\Phi(\tau, t)=\left[\begin{array}{cc}
\mathrm{I}_{3} & \Sigma(\tau, t) \\
0 & \mathrm{I}_{3}
\end{array}\right]
$$

where $\Sigma(\tau, t)=\frac{1}{2} \int_{t}^{\tau} Q_{1}\left(\tilde{\boldsymbol{q}}_{v}(\sigma)\right) I_{v}^{-1} R_{v}(\sigma) d \sigma$, with $Q_{1}\left(\tilde{\boldsymbol{q}}_{v}\right)$ defined in equation 7 . The observability Grammian is then ${ }^{10}$

$$
W(t, t+T)=\int_{t}^{t+T} \Phi(\tau, t)^{T} C^{T} C \Phi(\tau, t) d \tau=\int_{t}^{t+T}\left[\begin{array}{cc}
\frac{k}{2} \mathrm{I}_{3} & \frac{k}{2} \Sigma(\tau, t) \\
\frac{k}{2} \Sigma(\tau, t)^{T} & \frac{k}{2} \Sigma(\tau, t)^{T} \Sigma(\tau, t)
\end{array}\right] d \tau
$$

The system is UCO if there exists a $T>0$ and positive constants $\alpha_{1}<\infty, \alpha_{2}>0$ such that, for all $t \geq t_{0}, \alpha_{1} \mathrm{I} \geq W(t, t+T) \geq \alpha_{2} \mathrm{I}$. Using Lemma 13.4 of ref. 9 , this property is assured if $Q_{1}\left(\tilde{q}_{v}\right) I_{v}^{-1} R_{v}$ and $\frac{d}{d t}\left(Q_{1}\left(\tilde{\boldsymbol{q}}_{v}\right) I_{v}^{-1} R_{v}\right)$ are bounded, and there exist positive constants $T_{2}, \beta_{1}$, and a finite constant $\beta_{2}$ such that, for all $t \geq t_{0}$,

$$
\beta_{2} \mathrm{I}_{3} \geq \int_{t}^{t+T_{2}} R_{v}^{T} I_{v}^{-1} Q_{1}\left(\tilde{\boldsymbol{q}}_{v}\right)^{T} Q_{1}\left(\tilde{\boldsymbol{q}}_{v}\right) I_{v}^{-1} R_{v} d \tau \geq \beta_{1} \mathrm{I}_{3}
$$

$Q_{1}\left(\tilde{\boldsymbol{q}}_{v}\right) I_{v}^{-1} R_{v}$ is bounded, since all the signals in the estimator are bounded and the spacecraft inertia is bounded, hence the upper bound in 21 is satisfied. Substituting the equality $Q_{1}\left(\tilde{\boldsymbol{q}}_{v}\right)^{\mathrm{T}} Q_{1}\left(\tilde{\boldsymbol{q}}_{v}\right)=\mathrm{I}_{3}-\tilde{\varepsilon}_{v} \tilde{\varepsilon}_{v}^{T}$ into equation 21 results in

$$
\infty>\beta_{2} \mathrm{I}_{3} \geq \int_{t}^{t+T_{2}} R_{v}^{T} I_{v}^{-1}\left(\mathrm{I}_{3}-\bar{\varepsilon}_{v} \tilde{\varepsilon}_{v}^{T}\right) I_{v}^{-1} R_{v} d \tau \geq \beta_{1} \mathrm{I}_{3}>0
$$

Recall that it has been shown that $\left\|\tilde{\varepsilon}_{v}\right\| \rightarrow 0$ asymptotically. Thus, for any $\delta>0$, there exists a $T_{1}(\delta)>t_{0}$ such that $\left\|\tilde{\varepsilon}_{v}\right\|<\delta$ for all $t \geq t_{0}+T_{1}$. Taking any $\delta<1$, any $T_{2}>T_{1}$, and any $\boldsymbol{z}$ in $\mathbb{R}^{3}$

$$
\infty>\left(T_{2}-T_{1}\right) \lambda_{\text {max }}^{2}\|z\|^{2}>\boldsymbol{z}^{T}\left[\int_{t}^{t+T_{2}}\left(\mathrm{I}-\tilde{\varepsilon}_{v} \tilde{\epsilon}_{v}^{T}\right) d \tau\right] \boldsymbol{z}>\left(1-\delta^{2}\right)\left(T_{2}-T_{1}\right) \lambda_{\min }^{2}\|\boldsymbol{z}\|^{2}>0
$$

where $\lambda_{\max }$ and $\lambda_{\min }$ are the maximum and minimum eigenvalues of $I_{v}^{-1}$. Finally, $\frac{d}{d t} Q_{1}\left(\tilde{\boldsymbol{q}}_{v}\right) I_{v}^{-1} R_{v}$ is bounded, since all the terms in 15 are bounded. This demonstrates the required UCO property. The $\mathrm{PE}$ condition is satisfied, and therefore $\tilde{\varepsilon}_{v}$ and $\tilde{\boldsymbol{h}}_{i, v}$ approach zero exponentially fast, i.e $\left\|\hat{\boldsymbol{\omega}}_{v}\right\| \rightarrow\left\|\boldsymbol{\omega}_{v}\right\|$ exponentially fast.

\section{Error Sources}

The analysis provided in Section III is for the ideal scenario with no errors in any of the measurements needed for the estimator. In reality, the measurements will be corrupted by errors. The primary error source 
considered here is the error in the relative attitude measurement used to derive the HST attitude quaternion. The resulting error in the HST attitude quaternion leads also to an error in the computation of the gravity gradient torque, the dominant external torque acting on HST. The following analysis considers how the HST attitude error affects the estimator stability and convergence properties.

The measured HST quaternion, $\boldsymbol{q}_{v, m}$, is written as

$$
\boldsymbol{q}_{v, m}=\delta \boldsymbol{q}_{\text {err }} \otimes \boldsymbol{q}_{v}
$$

where $\delta \boldsymbol{q}_{e r r}$ is the error between the true HST quaternion, $\boldsymbol{q}_{v}$, and $\boldsymbol{q}_{\boldsymbol{v}, \boldsymbol{m}}$. Recall that $\boldsymbol{q}_{v}$ results from the product of the HRV relative attitude quaternion, $\boldsymbol{q}_{r}$, and the HRV inertial attitude quaternion, $\boldsymbol{q}_{h}$, shown in equation 9. The error is written as

$$
\boldsymbol{\delta} \boldsymbol{q}_{e r r}=\left[\begin{array}{l}
\varepsilon_{e r r} \\
\eta_{e r r}
\end{array}\right]
$$

From 24, the rotation matrices are given as

$$
R_{v, m}=R\left(\boldsymbol{q}_{v, m}\right)=R\left(\boldsymbol{\delta} \boldsymbol{q}_{e r r}\right) R\left(\boldsymbol{q}_{v}\right)=R\left(\boldsymbol{\delta} \boldsymbol{q}_{e r r}\right) R_{v}
$$

Multiplying both sides of equation 24 by $\hat{\boldsymbol{q}}_{v}$ gives

$$
\tilde{\boldsymbol{q}}_{v, m}=\boldsymbol{\delta} \boldsymbol{q}_{e r r} \otimes \tilde{\boldsymbol{q}}_{v}
$$

Similarly,

$$
R\left(\tilde{\boldsymbol{q}}_{v, m}\right)=R\left(\delta q_{e r r}\right) R\left(\tilde{\boldsymbol{q}}_{v}\right)
$$

Since the true quaternion is unknown, equations 13 and 14 cannot be implemented. Instead, $\boldsymbol{q}_{v, m}$ is used in place of $\boldsymbol{q}_{v}$, resulting in

$$
\begin{gathered}
\dot{\hat{\boldsymbol{q}}}_{v}=\frac{1}{2} Q\left(\hat{\boldsymbol{q}}_{v}\right) R\left(\tilde{\boldsymbol{q}}_{v, m}(t)\right)^{T}\left[I_{v}^{-1} R_{v, m} \hat{\boldsymbol{h}}_{i, v}+k \bar{\varepsilon}_{v, m} \operatorname{sign}\left(\tilde{\eta}_{v, m}\right)\right] \\
\dot{\hat{\boldsymbol{h}}}_{i, v}=\hat{T}_{i, v}+\frac{\alpha}{2} R_{v, m}^{T} I_{v}^{-1} \overline{\boldsymbol{\varepsilon}}_{v, m} \operatorname{sign}\left(\tilde{\eta}_{v, m}\right)
\end{gathered}
$$

$\hat{T}_{i, v}$ is the estimated external torque. Equations 25 and 27 are substituted into 28

$$
\dot{\hat{\boldsymbol{q}}}_{v}=\frac{1}{2} Q\left(\hat{\boldsymbol{q}}_{v}\right) R\left(\tilde{\boldsymbol{q}}_{v}\right)^{T}\left[R\left(\boldsymbol{\delta} \boldsymbol{q}_{e r r}\right)^{T} I_{v}^{-1} R\left(\boldsymbol{\delta} \boldsymbol{q}_{e r r}\right) R_{v} \hat{\boldsymbol{h}}_{i, v}+k R\left(\boldsymbol{\delta} \boldsymbol{q}_{e r r}\right)^{T} \tilde{\boldsymbol{\varepsilon}}_{v, m} \operatorname{sign}\left(\tilde{\eta}_{v, m}\right)\right]
$$

Rearranging the terms results in

$$
\dot{\hat{\boldsymbol{q}}}_{v}=\frac{1}{2} Q\left(\hat{\boldsymbol{q}}_{v}\right) R\left(\tilde{\boldsymbol{q}}_{v}\right)^{\mathrm{T}}\left[I_{v}^{-1} R_{v} \hat{\boldsymbol{h}}_{i, v}+k \tilde{\boldsymbol{\varepsilon}}_{v} \operatorname{sign}\left(\tilde{\eta}_{v}\right)+\Delta_{1} \hat{\boldsymbol{h}}_{i, v}+\Delta_{2}\right]
$$

where

$$
\begin{gathered}
\Delta_{1}=\left[R\left(\boldsymbol{\delta} \boldsymbol{q}_{e r r}\right)^{T} I_{v}^{-1} R\left(\boldsymbol{\delta} \boldsymbol{q}_{e r r}\right)-I_{v}^{-1}\right] R_{v} \\
\Delta_{2}=k\left[R\left(\delta \boldsymbol{q}_{e r r}\right)^{T} \tilde{\varepsilon}_{v, m} \operatorname{sign}\left(\bar{\eta}_{v, m}\right)-\tilde{\varepsilon}_{v} \operatorname{sign}\left(\tilde{\eta}_{v}\right)\right]
\end{gathered}
$$

$\Delta_{1}$ and $\Delta_{2}$ are both bounded. $\Delta_{1}$ results from the discrepancy in the HST inertia in the inertial frame due to the attitude error. $\Delta_{2}$ results directly from the attitude error.

Next, equation 29 is analyzed. Recall that the true angular momentum is driven by the external torques acting on HST, shown in equation 10. The dominant external torque acting on HST is gravity gradient torque. The gravity gradient torque in inertial coordinates is given as ${ }^{11}$

$$
T_{i, v}=\frac{3 \mu}{r_{i, v}^{3}} S\left(\boldsymbol{r}_{i, v}^{u}\right) R_{v}^{T} I_{v} R_{v} \boldsymbol{r}_{i, v}^{u}
$$

where $\mu$ is the earth's gravitational parameter, $r_{i, v}$ is the magnitude of the HST inertial position vector, and $r_{i, v}^{u}$ is the inertial position unit vector. The estimated torque in equation 29 is computed as

$$
\hat{T}_{i, v}=\frac{3 \mu}{r_{i, v}^{3}} S\left(r_{i, v}^{u}\right) R_{v, m}^{T} I_{v} R_{v, m} r_{i, v}^{u}
$$


Substituting equation 25 into $32, \hat{T}_{i, v}$ is written as

$$
\hat{T}_{i, v}=\frac{3 \mu}{r_{i, v}^{3}} S\left(r_{i, v}^{u}\right) R_{v}^{T} I_{v} R_{v} r_{i, v}^{u}+\Delta_{3}=T_{i, v}+\Delta_{3}
$$

where

$$
\Delta_{3}=\frac{3 \mu}{r_{i, v}^{3}} S\left(r_{i, v}^{u}\right)\left[R_{v, m}^{T} I_{v} R_{v, m}-R_{v}^{T} I_{v} R_{v}\right] r_{i, v}^{u}
$$

Substituting 33 and 25 into 29 results in

$$
\dot{\hat{h}}_{i, v}=T_{i, v}+\Delta_{3}+\frac{\alpha}{2} R_{v}^{T} I_{v}^{-1} \tilde{\varepsilon}_{v} \operatorname{sign}\left(\tilde{\eta}_{v}\right)+\Delta_{4}
$$

where

$$
\Delta_{4}=\frac{\alpha}{2} R_{v}^{T}\left[R\left(\boldsymbol{\delta} \boldsymbol{q}_{e r r}\right)^{T} I_{v}^{-1} \tilde{\varepsilon}_{v, m} \operatorname{sign}\left(\tilde{\eta}_{v, m}\right)-I_{v}^{-1} \dot{\tilde{\varepsilon}}_{v} \operatorname{sign}\left(\tilde{\eta}_{v}\right)\right]
$$

Combining equations 30 and 34 with 6 and 10 , we get

$$
\left[\begin{array}{c}
\dot{\tilde{\boldsymbol{q}}}_{v, m} \\
\tilde{\tilde{\boldsymbol{h}}} \\
\tilde{i}_{i, v}
\end{array}\right]=\left[\begin{array}{c}
\frac{1}{2} Q\left(\tilde{\boldsymbol{q}}_{v}(t)\right)\left(I_{v}^{-1} R_{v} \tilde{\boldsymbol{h}}_{i, v}-k \tilde{\boldsymbol{\varepsilon}}_{v} \operatorname{sign}\left(\tilde{\eta}_{v}\right)\right)+\Delta_{1}^{\prime} \hat{\boldsymbol{h}}_{i, v}+\Delta_{2}^{\prime} \\
-\frac{\alpha}{2} R_{v}^{T} I_{v}^{-1} \tilde{\varepsilon}_{v} \operatorname{sign}\left(\tilde{\eta}_{v}\right)+\Delta_{3}^{\prime}+\Delta_{4}^{\prime}
\end{array}\right]
$$

where $\Delta_{1}^{\prime}=-\frac{1}{2} Q\left(\tilde{\boldsymbol{q}}_{v}(t)\right) \Delta_{1}, \Delta_{2}^{\prime}=-\frac{1}{2} Q\left(\tilde{\boldsymbol{q}}_{v}(t)\right) \Delta_{2}, \Delta_{3}^{\prime}=-\Delta_{3}$, and $\Delta_{4}^{\prime}=-\Delta_{\mathbf{4}}$.

Equation 35 has the form

$$
\dot{\boldsymbol{x}}(t)=\boldsymbol{f}(t, \boldsymbol{x}(t))+\boldsymbol{d}(\boldsymbol{x}, t)
$$

where $f(\boldsymbol{x}, t)$ has been shown to be exponentially stable and

$$
d(t)=\left[\begin{array}{c}
\Delta_{1}^{\prime} \hat{h}_{i, v}+\Delta_{2}^{\prime} \\
\Delta_{3}^{\prime}+\Delta_{4}^{\prime}
\end{array}\right]=d_{1}(t)+d_{2}(t)
$$

where

$$
d_{1}(t)=\left[\begin{array}{c}
\Delta_{1}^{\prime} \hat{h}_{i, v} \\
0
\end{array}\right]
$$

and $d_{2}(t)$ contains the remaining terms, which are all bounded. Since $d_{2}(t)$ is bounded, the exponentially stable system is robust to this perturbation. The concern, however, is with $\boldsymbol{d}_{1}(t)$ which contains part of the state and hence cannot be assumed bounded a priori. If exponential stability is preserved with $d_{1}(t)$, then the system will be robust to the combined disturbance $d(t)$.

The nominal system is exponentially stable. Therefore, according to the Converse Lyapunov Theorem, a Lyapunov function and positive constants $c_{1}, c_{2}, c_{3}$, and $c_{4}$ exist for the nominal system and satisfy the following ${ }^{9}$

$$
\begin{gathered}
c_{1}\|\boldsymbol{x}(t)\| \leq V_{p}(t) \leq c_{2}\|\boldsymbol{x}(t)\| \\
\dot{V}_{p}(t) \leq-c_{3}\|\boldsymbol{x}(t)\|^{2} \\
\frac{\partial V_{p}(t)}{\partial x} \leq c_{4}\|x(t)\|
\end{gathered}
$$

Using $V_{p}(t)$ as the Lyapunov function candidate for the system perturbed by $d_{1}(t)$, the derivative of $V_{p}(t)$ satisfies

$$
\dot{V}_{p}(t) \leq-c_{3}\|x(t)\|^{2}+\left\|\frac{\delta V_{p}(t)}{\delta x}\right\|\left\|d_{1}(t)\right\|
$$

which becomes

$$
\dot{V}_{p}(t) \leq-c_{3}\|\boldsymbol{x}(t)\|^{2}+c_{4}\|\boldsymbol{x}(t)\|\left\|\boldsymbol{d}_{1}(t)\right\|
$$

Note that we can write

$$
\boldsymbol{d}_{1}(t)=\left[\begin{array}{c}
\Delta_{1}^{\prime} \hat{\boldsymbol{h}}_{i, v} \\
0
\end{array}\right]=\left[\begin{array}{c}
\Delta_{1}^{\prime}\left(\boldsymbol{h}_{i, v}-\tilde{\boldsymbol{h}}_{i, v}\right) \\
0
\end{array}\right]=\left[\begin{array}{c}
-\Delta_{1}^{\prime} \tilde{\boldsymbol{h}}_{i, v}+\Delta_{1}^{\prime} \boldsymbol{h}_{i, v} \\
0
\end{array}\right]
$$


$\Delta_{1}^{\prime} h_{i, v}$ can be moved into $d_{2}(t)$ since the angular momentum of HST will be bounded. Equation 36 is then

$$
\begin{gathered}
\dot{V}_{p}(t) \leq-c_{3}\|x(t)\|^{2}+c_{4}\|x(t)\|\left\|\left[\begin{array}{c}
-\Delta_{1}^{\prime} \tilde{h}_{i, v} \\
0
\end{array}\right]\right\| \\
\dot{V}_{p}(t) \leq-c_{3}\|x(t)\|^{2}+c_{4} \delta\|x(t)\|^{2}
\end{gathered}
$$

where $\left\|\Delta_{1}^{\prime}\right\| \leq \delta$. If $\delta$ is sufficiently small, exponential stability is preserved, and the system is robust to the combined perturbation $d(t)=d_{1}(t)+d_{2}(t)$

Adding $d_{2}(t)$ back into the Lyapunov analysis, equation 37 becomes

$$
\dot{V}_{p}(t) \leq-c_{3}\|x(t)\|^{2}+c_{4} \delta\|x(t)\|^{2}+c_{4}\|x(t)\|\left\|d_{2}(t)\right\|
$$

The bound on $\left\|\Delta_{1}^{\prime}\right\|$, which establishes the size of $\delta$, and the bound on $\left\|d_{2}(t)\right\|$ are defined next. From equation $2, R\left(\delta q_{\text {err }}\right)$ can be written as

$$
\begin{aligned}
R\left(\delta q_{e r r}\right) & =\left(\eta_{e r r}^{2}-\varepsilon_{e r r}^{T} \varepsilon_{e r r}\right) \mathrm{I}_{3}+2 \varepsilon_{e r r} \varepsilon_{e r r}^{T}-2 \eta_{e r r} S\left(\varepsilon_{e r r}\right)=\mathrm{I}_{3}-2 \varepsilon_{e r r}^{T} \varepsilon_{e r r}+2 \varepsilon_{e r r} \varepsilon_{e r r}^{T}-2 \eta_{e r r} S\left(\varepsilon_{e r r}\right) \\
& =\mathrm{I}_{3}+Y\left(\eta_{e r r}, \varepsilon_{e r r}\right)
\end{aligned}
$$

and

$$
\left\|Y\left(\eta_{e r r}, \varepsilon_{e r r}\right)\right\|=2\left\|\varepsilon_{e r r}\right\|
$$

Also, note that $\left\|Q\left(\tilde{q}_{v}(t)\right)\right\|=1,\left\|R_{v}\right\|=\left\|R\left(\tilde{\boldsymbol{q}}_{v}\right)\right\|=1,\left\|\tilde{\varepsilon}_{v}\right\| \leq 1,\left\|\tilde{\varepsilon}_{v, m}\right\| \leq 1,\left\|I_{v}\right\| \leq \lambda_{\max }$, and $\left\|I_{v}^{-1}\right\| \leq$ $\frac{1}{\lambda_{\min }}$. The $\left\|\Delta_{1}^{\prime}\right\|$ is then bounded as

$$
\left\|\Delta_{1}^{\prime}\right\| \leq \frac{1}{2}\left\|\Delta_{1}\right\| \leq \frac{2\left\|\varepsilon_{\text {err }}\right\|}{\lambda_{\min }}\left(1+\left\|\varepsilon_{\text {err }}\right\|\right) \leq \delta
$$

The $\left\|d_{2}(t)\right\|$ is bounded as

$$
\left\|d_{2}(t)\right\| \leq\left\|\Delta_{2}^{\prime}\right\|+\left\|\Delta_{3}^{\prime}\right\|+\left\|\Delta_{4}^{\prime}\right\|+\left\|\Delta_{1}^{\prime}\right\|\left\|h_{i, v}\right\|_{\max }
$$

with

$$
\begin{gathered}
\left\|\Delta_{2}^{\prime}\right\| \leq k\left\|\varepsilon_{e r r}\right\| \\
\left\|\Delta_{3}^{\prime}\right\| \leq \frac{12 \mu \lambda_{\text {max }}\left\|\varepsilon_{e r r}\right\|}{r_{i, v}^{3}}\left(1+\left\|\varepsilon_{e r r}\right\|\right) \\
\left\|\Delta_{4}^{\prime}\right\| \leq \frac{\alpha}{\lambda_{\text {min }}}\left\|\varepsilon_{\text {err }}\right\|
\end{gathered}
$$

The angular momentum of HST is bounded as

$$
\left\|\boldsymbol{h}_{i, v}\right\|=\left\|\boldsymbol{h}_{b, v}\right\| \leq \lambda_{\max }\left\|\boldsymbol{\omega}_{b, v, \max }\right\|
$$

Extensive simulations of HST predict the maximum angular velocity as approximately $0.004 \mathrm{rad} / \mathrm{sec}^{12}$ Therefore,

$$
\left\|\Delta_{1}^{\prime}\right\|\left\|\boldsymbol{h}_{i, v}\right\|_{\max } \leq \frac{0.008 \lambda_{\max }\left\|\varepsilon_{e r r}\right\|}{\lambda_{\min }}\left(1+\left\|\varepsilon_{e r r}\right\|\right)
$$

Applying Young's inequality to equation 38, and combining terms gives

$$
\dot{V}_{p}(t) \leq-\left(\frac{1}{2} c_{3}-c_{4} \delta\right)\|x(t)\|^{2}+\frac{c_{4}^{2}}{2 c_{3}}\left\|d_{2}(t)\right\|^{2}
$$

Substituting all the bounds into equation 40 , equation 41 is written as

$$
\dot{V}_{p}(t) \leq-\left(\frac{1}{2} c_{3}-c_{4} \delta\right)\|x(t)\|^{2}+\frac{c_{4}^{2}}{2 c_{3}}\left\|\varepsilon_{e r r}\right\|^{2}\left(a+b\left\|\varepsilon_{e r r}\right\|\right)^{2}
$$


where $a=k+\frac{\alpha}{\lambda_{\min }}+\frac{12 \mu \lambda_{m} a x}{r_{i, v}^{3}}+\frac{0.008 \lambda_{m} a x}{\lambda_{\min }}$ and $b=\frac{12 \mu \lambda_{m} a x}{r_{i, v}^{3}}+\frac{0.008 \lambda_{m} a x}{\lambda_{\min }}$. Recall that $\left\|\varepsilon_{e r r}\right\|=\sin \frac{1}{2} \phi_{e r r}$. Assuming the $\phi_{\text {err }}$ is small, $\left\|\varepsilon_{\text {err }}\right\| \approx \frac{1}{2} \phi_{\text {err }}$. Equation 42 is then

$$
\dot{V}_{p}(t) \leq-\left(\frac{1}{2} c_{3}-c_{4} \delta\right)\|x(t)\|^{2}+\frac{c_{4}^{2}}{8 c_{3}} \phi_{e r r}^{2}\left(a+\frac{1}{2} b \phi_{e r r}\right)^{2}
$$

Neglecting powers of $\phi_{\text {err }}$ greater than 2 gives

$$
\dot{V}_{p}(t) \leq-\left(\frac{1}{2} c_{3}-c_{4} \delta\right)\|\boldsymbol{x}(t)\|^{2}+\frac{a^{2} c_{4}^{2}}{8 c_{3}} \phi_{e r r}^{2}
$$

Computing the time average of equation 44 , and taking the limit as $T \rightarrow \infty$, is

$$
\limsup _{T \rightarrow \infty} \frac{1}{T} \int_{0}^{T}\|x(\tau)\|^{2} d \tau \leq \limsup _{T \rightarrow \infty} \frac{a^{2} c_{4}^{2}}{8 c_{3}\left(c_{3}-c_{4} \delta\right)} \int_{0}^{T} \phi_{e r r}(\tau)^{2} d \tau
$$

If the error process is ergodic, the ensemble average is equivalent to the time average. The RMS bound is given as

$$
\left\|\tilde{\boldsymbol{h}}_{i, v}\right\|_{R M S} \leq\|\boldsymbol{x}(t)\|_{R M S} \leq c \sigma
$$

where $\sigma$ is the standard deviation of the error process, and all the constants are combined together as $c=\frac{a c_{4}}{8 c_{3}\left(c_{3}-c_{4} \delta\right)}$.

Equation 45 serves to demonstrate that the angular momentum estimator is bounded, and converges to a ball sized by the bound given in 45 (given that the attitude is corrupted by errors meeting the above statistical assumptions). Equation 45 sizes the errors only to within the original unknown constants of the converse Lyapunov analysis. The characteristics of the estimator error will be addressed through numerical simulations.

The instability resulting from $d_{1}(t)$ can be removed through the introduction of a 'leakage' term in equation 29.

$$
\dot{\hat{\boldsymbol{h}}}_{i, v}=\hat{T}_{i, v}+\frac{\alpha}{2} R_{v, m}^{T} I_{v}^{-1} \tilde{\varepsilon}_{v, m} \operatorname{sign}\left(\tilde{\eta}_{v, m}\right)-\sigma\left(\hat{\boldsymbol{h}}_{i, v}\right) \hat{\boldsymbol{h}}_{i, v}
$$

where

$$
\sigma\left(\hat{\boldsymbol{h}}_{i, v}\right)= \begin{cases}\sigma_{o} & \text { if }\left\|\hat{\boldsymbol{h}}_{i, v}\right\|>\left\|\boldsymbol{h}_{i, v}\right\|_{\max }>\left\|\boldsymbol{h}_{i, v}\right\| \\ 0 & \text { otherwise }\end{cases}
$$

where the scalar $\sigma_{o}>0$ and $\left\|\boldsymbol{h}_{i, v}\right\|_{\text {max }}$ is the upper bound on the angular momentum established above.

The additional term in 46 introduces an additional term into equation 38

$$
\sigma \tilde{h}_{i, v}^{T} \hat{\boldsymbol{h}}_{i, v}=\sigma \tilde{h}_{i, v}\left(\boldsymbol{h}_{i, v}-\tilde{h}_{i, v}\right)=-\sigma\left\|\tilde{h}_{i, v}\right\|^{2}+\sigma \tilde{h}_{i, v}^{T} \boldsymbol{h}_{i, v}
$$

Applying Young's inequality results in

$$
\sigma \tilde{h}_{i, v}^{T} \hat{\boldsymbol{h}}_{i, v} \leq-\frac{1}{2} \sigma\left\|\tilde{h}_{i, v}\right\|^{2}+\frac{1}{2} \sigma\left\|\boldsymbol{h}_{i, v}\right\|^{2}
$$

Since $h_{i, v}$ is assumed to be bounded, the second term acts as another bounded disturbance. The first term, however, acts to stabilize the possible high noise instability identified above. In particular, if $\sigma_{o}>2 c_{4} \delta$ then the system remains stable. The process to establish the bounds outlined above remains the same, with $c_{3}-c_{4} \delta+\frac{1}{2} \sigma_{o}$ replacing $c_{3}-c_{4} \delta$.

Although it looks like this technique adds an additional disturbance, possibly stabilizing the system but adding an extra error, in fact this is not the case. Expanding the added term instead as

$$
\sigma \tilde{\boldsymbol{h}}_{i, v} \hat{\boldsymbol{h}}_{i, v}=\sigma\left(\boldsymbol{h}_{i, v}-\hat{\boldsymbol{h}}_{i, v}\right)^{T} \hat{\boldsymbol{h}}_{i, v}=\sigma \boldsymbol{h}_{i, v} \hat{\boldsymbol{h}}_{i, v}-\sigma\left\|\hat{\boldsymbol{h}}_{i, v}\right\|^{2}
$$

Or

$$
\sigma \tilde{h}_{i, v} \hat{\boldsymbol{h}}_{i, v} \leq \frac{1}{2} \sigma\left[\left\|\boldsymbol{h}_{i, v}\right\|^{2}-\left\|\hat{\boldsymbol{h}}_{i, v}\right\|^{2}\right]
$$

Recall from above the $\sigma>0$ only if $\left\|\hat{\boldsymbol{h}}_{i, v}\right\|>\left\|\boldsymbol{h}_{i, v}\right\|$. Thus the added term is always negative; essentially the extra constraint in the adaptation law can only help convergence. 


\section{HST Pseudo-Linear Angular Velocity Estimator}

The pseudo-linear Kalman filter is based on the model presented in reference 13. A brief overview of the filter models is given first, followed by a summary of the actual algorithm equations.

The state of the filter is

$$
\boldsymbol{X}=\left[\begin{array}{c}
\boldsymbol{q}_{v} \\
\boldsymbol{\omega}_{v}
\end{array}\right]
$$

where $\boldsymbol{q}_{v}$ is the HST inertial attitude and $\boldsymbol{\omega}_{v}$ is the HST angular velocity in HST body coordinates. The quaternion is propagated according to equation 6 as

$$
\dot{\boldsymbol{q}}_{v}=\frac{1}{2} Q\left(\boldsymbol{q}_{v}\right) \boldsymbol{\omega}_{v}
$$

The angular velocity is propagated using Euler's equation as

$$
\dot{\boldsymbol{\omega}}_{v}=I_{v}^{-1}\left[S\left(I_{v} \boldsymbol{\omega}_{v}\right) \boldsymbol{\omega}_{v}+T_{b, v}\right]
$$

where $T_{b, v}$ is the gravity gradient torque from equation 32 , transformed to body coordinates as

$$
T_{b, v}=\frac{3 \mu}{r_{i, v}^{3}} S\left(\boldsymbol{r}_{b, v}^{u}\right) I_{v} R_{v} \boldsymbol{r}_{i, v}^{u}
$$

where $\boldsymbol{r}_{b, v}^{u}=R_{v} \boldsymbol{r}_{i, v}^{u}$. Equation 49 can be written in a pseudo-linear form as

$$
T_{b, v}=\frac{3 \mu}{r_{i, v}^{3}} S\left(r_{b, v}^{u}\right) I_{v} M\left(\boldsymbol{r}_{i, v}^{u}, \boldsymbol{q}_{v}\right) \boldsymbol{q}_{v}=F_{g g} \boldsymbol{q}_{v}
$$

where $M\left(\boldsymbol{r}_{i, v}^{u}, \boldsymbol{q}_{v}\right)$ is defined in reference 14. Equation 50 is substituted into 48 , and equations 47 and 48 are then augmented as

$$
\dot{\boldsymbol{X}}=F(\boldsymbol{X}) \boldsymbol{X}+\boldsymbol{w}(t)
$$

where $w(t)$, a zero-mean white noise process, is added to account for the lack of knowledge in the terms given in equations 47 through $50 . F(\boldsymbol{X})$ is defined as

$$
F(\boldsymbol{X})=\left[\begin{array}{cc}
0 & \frac{1}{2} Q\left(\boldsymbol{q}_{v}\right) \\
I_{v}^{-1} F_{g g} & I_{v}^{-1} S\left(I_{v} \boldsymbol{\omega}_{v}\right)
\end{array}\right]
$$

The measurement used in the filter is $\boldsymbol{q}_{v, m}$ given in equation 24 . The measurement model for a given time $t_{k}$ is simply

$$
\boldsymbol{q}_{v, m, k}=\left[\begin{array}{ll}
\mathrm{I}_{\mathbf{4}} & 0
\end{array}\right] \boldsymbol{X}_{k}=H \boldsymbol{X}_{k}+\boldsymbol{v}_{k}
$$

where $\mathrm{I}_{4}$ is a $4 \times 4$ identity matrix and $v_{k}$ is a zero-mean white measurement noise.

The filter algorithm is implemented discretely as follows:

Propagation:

$$
\begin{gathered}
\hat{\boldsymbol{X}}_{k+1}(-)=\Phi_{k} \hat{\boldsymbol{X}}_{k}(+) \\
\Phi_{k}=\mathrm{e}^{F\left(\hat{\boldsymbol{X}}_{k}(+)\right) \Delta t} \\
\Delta t=t_{k+1}-t_{k} \\
P_{k+1}(-)=\Phi_{k} P_{k}(+) \Phi_{k}^{T}+Q
\end{gathered}
$$

where $P$ is the filter covariance, $Q$ is the process noise covariance matrix, and $(-)$ and $(+)$ indicate a priori and a posteriori values, respectively.

Update:

$$
\begin{gathered}
\hat{\boldsymbol{X}}_{k}(+)=\hat{\boldsymbol{X}}_{k}(-)+K_{k}\left(\boldsymbol{q}_{v, m, k}-\hat{\boldsymbol{q}}_{k}(-)\right) \\
K_{k}=H P_{k}(-)\left(H P_{k}(-) H^{T}+R\right)^{-1} \\
P_{k}(+)=\left(\mathrm{I}-K_{k} H\right) P_{k}(-)\left(\mathrm{I}-K_{k} H\right)^{T}+K_{k} R K_{k}^{T}
\end{gathered}
$$

where $K_{k}$ is the filter gain matrix at time, $t_{k}$, and $\mathrm{R}$ is the measurement noise covariance matrix. 


\section{Simulation Results}

The algorithms outlined in sections III through $\mathrm{V}$ are tested with a simulation. The simulation includes two orbits of data, based on an actual HST ephemeris. The HST inertia is ${ }^{12}$

$$
I_{v}=\left[\begin{array}{ccc}
36046 & -706 & 1491 \\
-706 & 86868 & 449 \\
1491 & 449 & 93848
\end{array}\right] \mathrm{kg} \cdot \mathrm{m}^{2}
$$

Both algorithms are initially tested without any errors. In both cases, the initial attitude quaternions are identity, $\boldsymbol{q}_{v}=\hat{\boldsymbol{q}}_{v}=[0,0,0,1]$. The initial HST angular velocity estimate is zero, $\hat{\boldsymbol{\omega}}_{v}=[0,0,0]$, and the true initial angular velocity is $\omega_{v}=[-0.04,-0.01,0.14] \mathrm{deg} / \mathrm{sec}$.

Figures 1 and 2 are examples of the resulting angular velocity error from the nonlinear estimator and the pseudo-linear filter, respectively. The pseudo-linear algorithm converges very quickly, but the error oscillates. The nonlinear estimator has a longer convergence time, however the errors after convergence are very small.

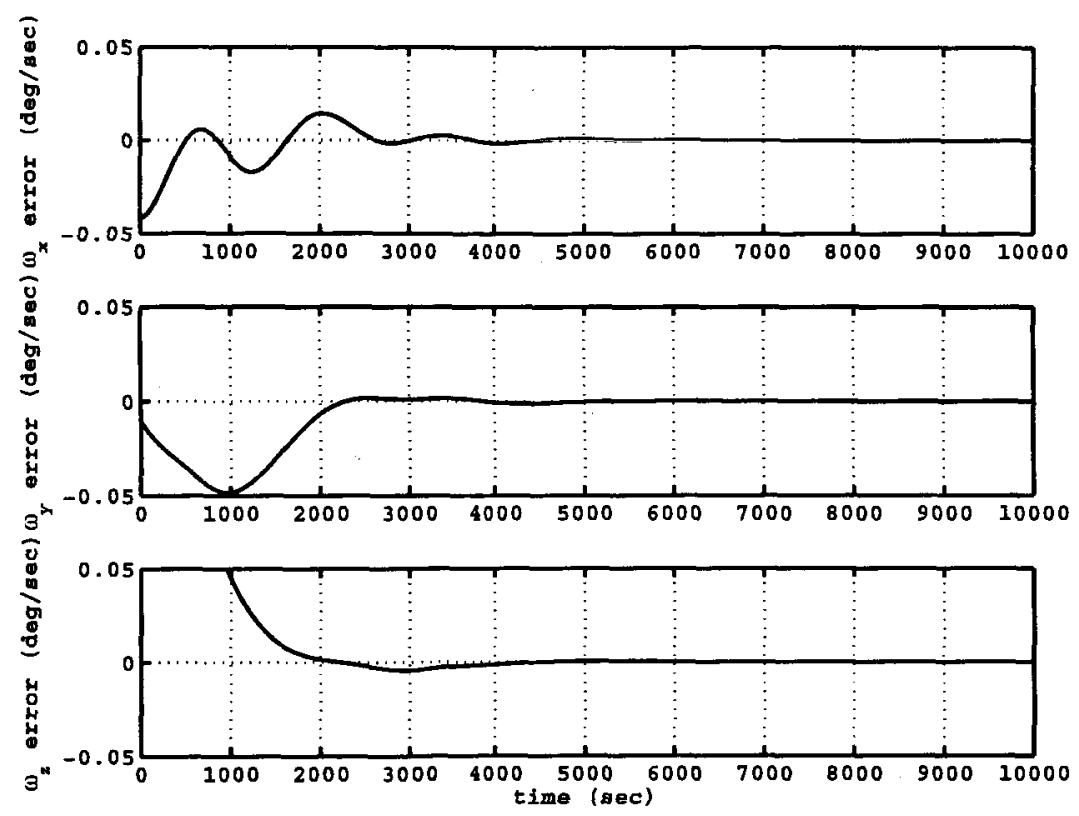

Figure 1. Angular Velocity Errors: Nonlinear, No Attitude Measurement Errors 


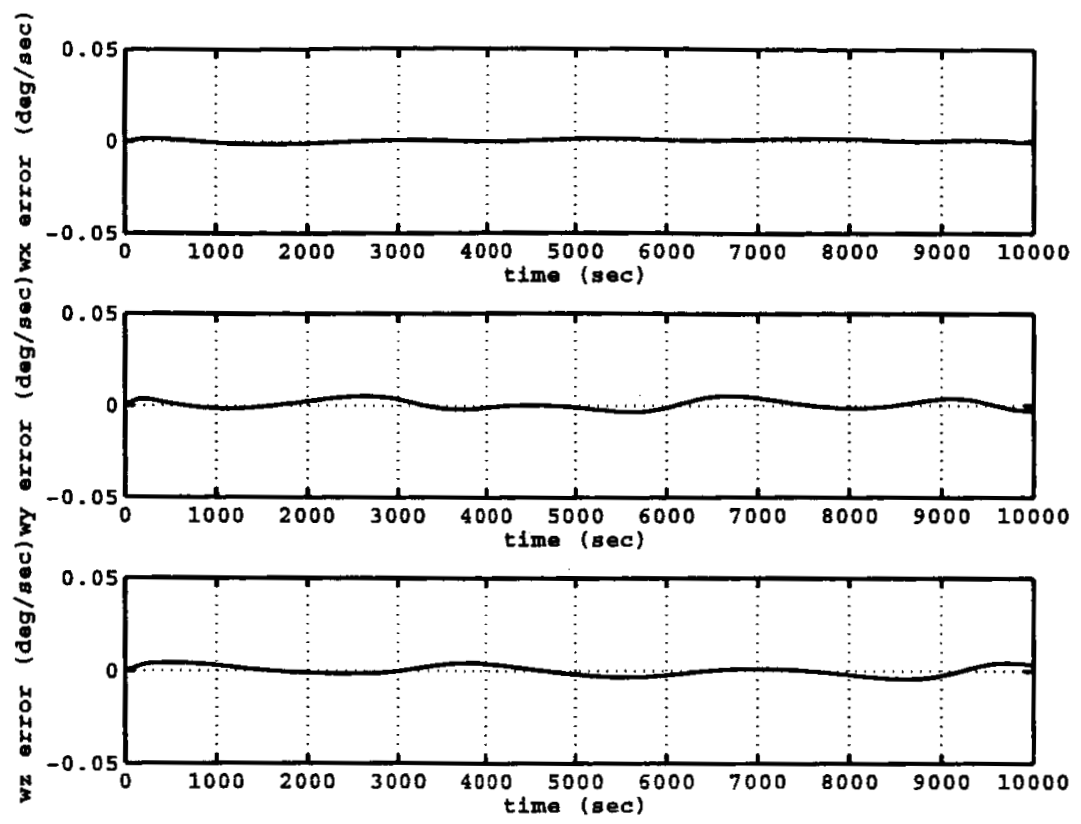

Figure 2. Angular Velocity Errors: Pseudo-Linear, No Attitude Measurement Errors

Next, the measured attitude is chosen randomly with a 15 degree uncertainty. Figures 3 and 4 are samples of the angular velocity error for the nonlinear estimator and the pseudo-linear filter, respectively, using the same erroneous measured attitudes. As with the clean example above, the pseudo-linear filter converges faster, but is noisier than the nonlinear estimator in steady state.

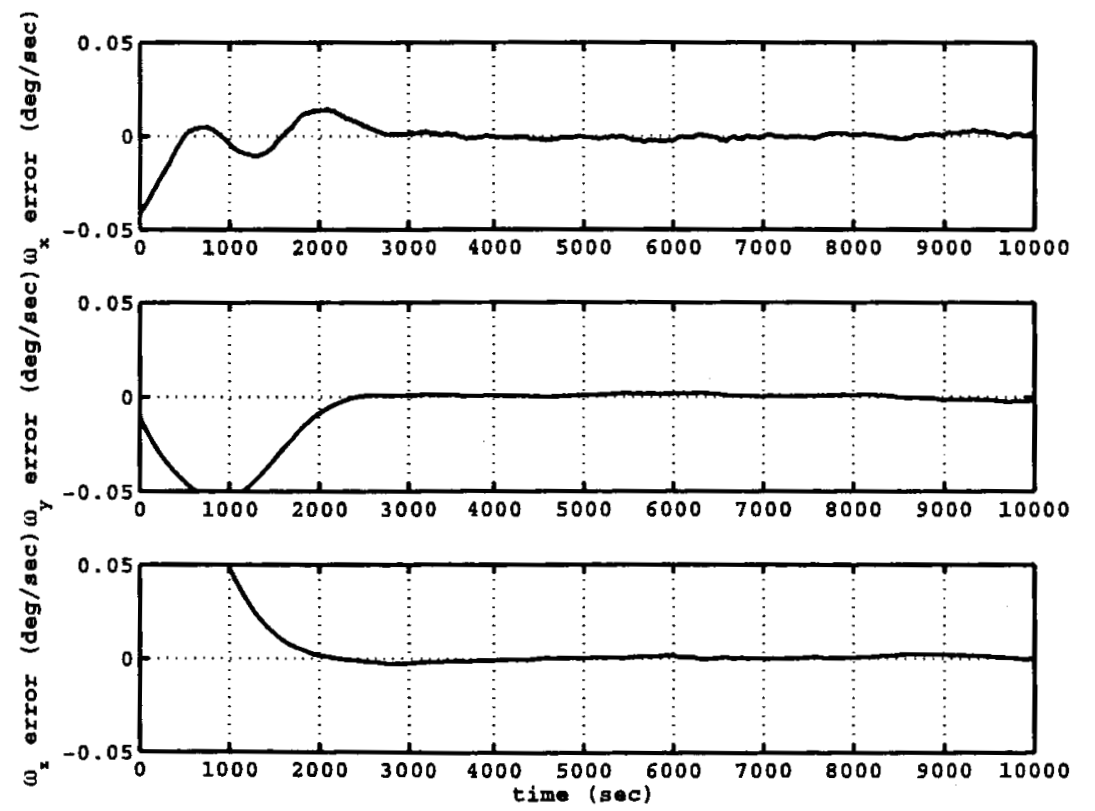

Figure 3. Sample Angular Velocity Error: Nonlinear, Random 15 Degree Measured Attitude Error

Table 1 lists the average of the final RMS angular velocity error for each of the 100 test cases, for both the nonlinear estimator and the pseudo-linear Kalman filter. The average errors are smailer for the nonlinear 


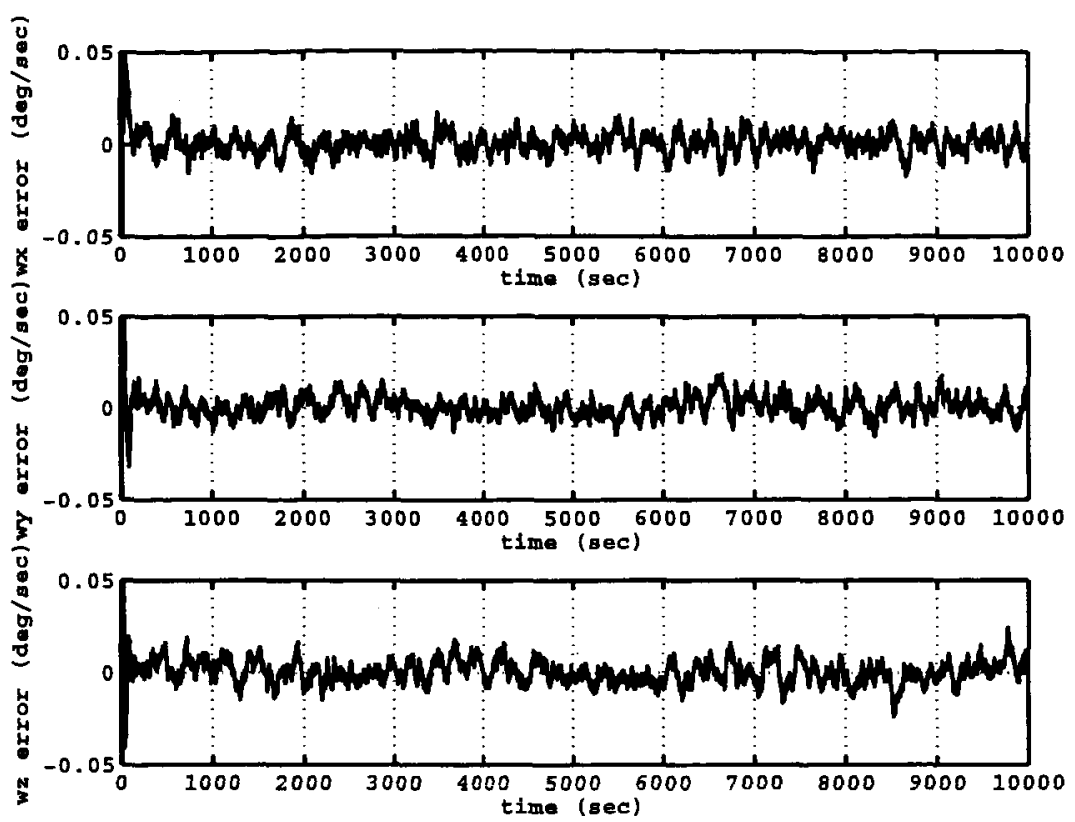

Figure 4. Sample Angular Velocity Error: Pseudo-linear, Random 15 Degree Measured Attitude Error

estimator on all three axes.

Table 1. Average Final RMS Angular Velocity Errors

\begin{tabular}{|l|c|c|}
\hline & Nonlinear $(\mathrm{deg} / \mathrm{sec})$ & Pseudo-Linear $(\mathrm{deg} / \mathrm{sec})$ \\
\hline$\tilde{\omega}_{x, R M S}$ & 0.00164 & 0.00516 \\
\hline$\tilde{\omega}_{y, R M S}$ & 0.00164 & 0.00558 \\
\hline$\tilde{\omega}_{z, R M S}$ & 0.00127 & 0.00555 \\
\hline
\end{tabular}

Finally, figure 5 shows the RMS angular momentum at the end of two orbits for each of the 100 test cases. These results could be utilized in analyzing the numerical bound expressed in equation 45 .

\section{Conclusions}

A nonlinear algorithm is developed to estimate the rotation rates for a 'non-cooperative' target vehicle. The nonlinear algorithm determines the rotation rate through an estimation of the inertial angular momentum. The algorithm, designed for the Hubble Robotic Servicing mission, applies in particular to the scenario in which the batteries have died and HST is tumbling. The HRV design includes vision and feature recognition sensors capable of producing a relative attitude quaternion. Combining the relative attitude with the HRV inertial attitude produces the measured HST attitude for the estimation algorithm. The nonlinear algorithm is compared to a pseudo-linear Kalman filter.

The two rate estimation approaches are compared using two orbits of HST data. Initially, the algorithms are compared with perfect measured attitudes. Both algorithms estimate the HST rotation rate. The pseudo-linear algorithm converges quickly, but oscillates slightly in steady state. The nonlinear approach is slower to converge; the steady state errors, however, do not oscillate and are very nearly zero. The two approaches are then compared for 100 different cases, with different random measured attitudes in each case. Again, both approaches estimate the rotation rate quite well. However, the nonlinear approach produces consistently smaller final rotation rate errors. 


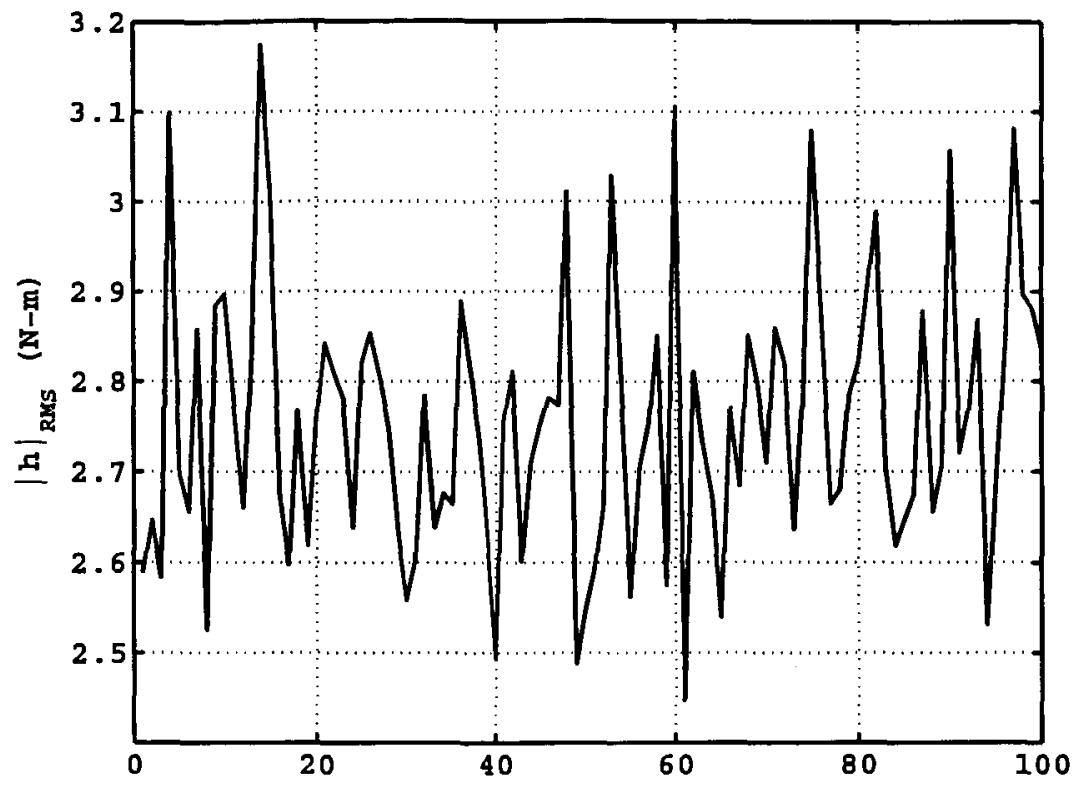

Figure 5. Final Angular Momentum Error RMS

Future work will focus on improving the accuracy of the relative attitude quaternion computed from the vision and feature recognition sensors. Additional error sources, such as uncertainties in the spacecraft inertia, should be addressed, as well as a more rigorous study of the nonlinear estimator gains. Also, the algorithms will be coupled with a control scheme to study the closed loop behavior during a rendezvous or docking scenario.

\section{References}

${ }^{1}$ Shuster, M. D., "A Survey of Attitude Representations," The Journal of Astronautical Sciences, Vol. 41, No. 4, OctoberDecember 1993, pp. 439-517.

${ }^{2}$ Sanner, R. M., "Adaptive Attitude Control Using Fixed and Dynamically Structured Neural Networks," AIAA Guidance, Navigation, and Control Conference, No. 96-3891, San Diego, California, July 1996.

${ }^{3}$ Wie, B., Space Vehicle Dynamics and Control, AIAA Education Series, 1998.

${ }^{4}$ Salcudean, S., "A Globally Convergent Angular Velocity Observer for Rigid Body Motion," IEEE Transactions on Automatic Control, Vol. 36, No. 12, December 1991, pp. 1493-1496.

${ }^{5}$ Thienel, J., Nonlinear Observer/Controller Designs for Spacecraft Attitude Control Systems with Uncalibrated Gyros, Ph.D. thesis, University of Maryland, 2004.

${ }^{6}$ Thienel, J. and Sanner, R. M., "A Globally Convergent Angular Velocity Observer for Rigid Body Motion," IEEE Transactions on Automatic Control, Vol. 48, No. 11, November 2003, pp. 2011-2015.

${ }^{7}$ Bar-Itzhack, I., "Navigation Computation in Terrestrial Strapdown Inertial Navigation Systems," IEEE Transactions on Aerospace and Electronic Systems, Vol. AES-13, No. 6, November 1977, pp. 679-689.

${ }^{8}$ Krstić, M., Kanellakopoulos, I., and Kokotovic, P., Nonlinear and Adaptive Control Design, John Wiley and Sons, Inc., 1995.

${ }^{9}$ Khalil, H. K., Nonlinear Systems, Prentice-Hall, Inc., 2nd ed., 1996.

${ }^{10}$ Chen, C.-T., Linear System Theory and Design, Oxford University Press, 2nd ed., 1984.

${ }^{11}$ Wertz, J. R., editor, Spacecraft Attitude Determination and Control, D. Reidel Publishing Company, 1984.

${ }^{12}$ Queen, S., "HRV GNC Peer Review, Flight Performance Analysis," Tech. rep., NASA Goddard Space Flight Center, 2004.

${ }^{13}$ Azor, R., Bar-Itzhack, I., Harman, R., and Deutschmann, J., "Angular Rate Estimation Using Delayed Quaternion Measurements," AIAA Journal of Guidance, Control, and Dynamics, Vol. 24, No. 3, May-June 2001, pp. 436-443.

${ }^{14}$ Bar-Itzhack, I. and Harman, R., "Pseudo-Linear Attitude Determination for Spinning Spacecraft," AIAA/AAS Astrodynamics Specialist Conference, No. AIAA-2004-5387, Providence, Rhode Island, August 2004 